\title{
Characterization of materials for an embankment of highway by plate bearing test with Plaxis modelling (Case East/West Motorway)
}

\author{
Belouar Abdelghani ${ }^{1}$, Ykhlef Boubakeur ${ }^{1}$ et Boubidi Sayed ${ }^{1}$ \\ ${ }^{1}$ Université Constantine 1 LMDC, Department of civil Engineering, Constantine, Algeria
}

\begin{abstract}
This paper aims to characterize materials used in the embankment for the highway, namely the identification and analysis of mechanical characteristics of materials which would be used in the construction of the East/West Motorway, section Constantine-Annaba (experimental study). However, the tests were carried out in geotechnical laboratory of the University of Constantine1. Then, for validating the results obtained in experiments, a numerical modelling of the plate bearing test by a finite element method (FEM) has been carried out using "PLAXIS" software. The numerical results obtained are in good agreement with those of the experimental.
\end{abstract}

\section{Introduction}

L'objectif de cette communication est l'identification ainsi que l'analyse des caractéristiques mécaniques des matériaux utilisés dans la réalisation d'un tronçon d'autoroute Est/Ouest Constantine-Annaba, suivie d'une modélisation numérique de l'essai de plaque par la méthode des éléments finis à l'aide du logiciel Plaxis 2D.

Le fonctionnement d'une chaussée en général doit être soumis aux règles fondamentales pour concevoir et dimensionner une route, mais aussi les spécifications requises pour les matériaux routiers, et le soin à apporter à leur mise en œuvre [1]. Le poids du véhicule est transmis au sol, sous forme de pressions, par l'intermédiaire des pneumatiques. D'une manière générale, les sols ne peuvent supporter sans dommage de telles pressions. Si le sol n'est pas assez porteur, le pneu comprime le sol et il se forme une ornière, Dans une couche granulaire non liée, les grains constitutifs restent indépendants les uns des autres.

Sous l'action d'une charge, cette couche travaille principalement en compression, c'est-à-dire qu'elle transmet au sol sous-jacent la totalité de la charge en la répartissant d'une façon non uniforme. La pression la plus importante se situe en dessous de la charge.

Mais, à l'interface couche granulaire-sol, on peut constater que la somme des charges réparties est égale à la charge $\mathrm{P}$. La pression la plus élevée se situe alors à la verticale de la charge. Pour dimensionner une couche granulaire, c'est-à-dire pour définir son épaisseur, il faut que la pression verticale maximale transmise au sol sousjacent soit inférieure à la portance du sol [2].

\section{Essai de plaque}

Cet essai est utilisé lors des travaux de terrassement et de fondation. Elle est applicable pour le contrôle du compactage des sols ainsi que comme base pour le dimensionnement à la portance des routes, des voies ferrées, des places d'aviation et d'autres ouvrages [3].

L'essai de plaque permet de déterminer des courbes charge-tassement, afin d'évaluer la déformabilité et la portance des sols. Les modules de déformation EV1, correspondant à un premier chargement, et EV2, correspondant à un deuxième chargement, peuvent être détermines à partir des courbes charge-tassement [4].

Les différents points de mesure des cycles de chargement et de déchargement seront représentés par une courbe lissée figure 1 .

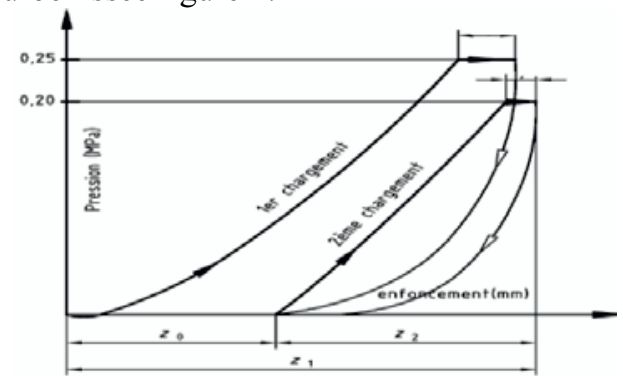

Fig. 1. Courbe charge-tassement durant l'essai de plaque [3]

Les courbes sont définies par un polynôme du deuxième degré dont les constantes seront tirées des valeurs mesurées par la méthode des moindres carres (régression) [4].

$S=a_{0}+a_{1} \sigma+a_{2} \sigma^{2}$

$\sigma:$ contrainte normale moyenne sous la plaque,

$\mathrm{S}$ : tassement, a0, a1, a2 : constantes du polynôme. 


\section{Méthode théorique}

\subsection{Théories de l'élasticité}

L'une des fonctions principales de chaussée est de distribuer la charge induite par le trafic. Par souci de simplicité, la plupart des méthodes de calcul de la réponse mécanique de la chaussée sont basées sur des hypothèses simplificatrices (charge idéalisée, comportement linéaire élastique des matériaux et couches constituées de matériaux homogènes et isotropes).

Boussinesq calcule la distribution des contraintes et déformations dans un massif uniforme sous une charge ponctuelle ou distribuée sur une plaque souple [4].

\subsection{Modèle de Boussinesq}

Boussinesq calcule les contraintes, les déformations, et les déflexions verticales (sous le centre de la plaque) à la profondeur (z), sur une plaque de rayon (r) [4]

$$
\begin{aligned}
& \sigma_{1}=\sigma_{0}\left[1-\frac{\mathrm{z}^{3}}{\left(r^{2}+z^{2}\right)^{\frac{3}{2}}}\right] \\
& \varepsilon_{1}=\frac{(1+\mu) \sigma_{0}}{E}\left[(1-2 \mu)+\frac{2 \mu \mathrm{z}}{\left(r^{2}+z^{2}\right)^{\frac{1}{2}}}-\frac{z^{3}}{\left(r^{2}+z^{2}\right)^{\frac{3}{2}}}\right] \\
& d_{1}=\frac{k\left(1-\mu^{2}\right) \sigma_{0} r}{E}
\end{aligned}
$$

$\mathrm{k}$ : coefficient $=2$ pour une plaque articulée et $\pi / 2$ pour une plaque rigide ; $\mu$ : coefficient de poisson ;

$\sigma_{0}$ : contrainte de compression sous la plaque $(\mathrm{MPa})$

$\mathrm{r}$ : rayon de la plaque $(\mathrm{m}) ; \mathrm{d} 0$ : déflexion $(\mathrm{m})$.

Le calcul du module élastique de Boussinesq pour une charge circulaire est base sur la déflexion centrale $d_{0}$ comme le montre la formule suivante :

$$
E=k \frac{\left(1-v^{2}\right) \cdot \sigma_{0} \cdot r}{d_{0}}(\mathrm{MPa})
$$

Le module de compressibilité EV est l'homologue d'un module d'élasticité [4], c'est donc une caractéristique du sol. Ce module est relie directement avec le module d'élasticité $E$ et le coefficient de poisson $v$ par la formule suivante :

$E=\frac{\pi}{4}\left(1-v^{2}\right) E V$

Le module de compressibilité varie pratiquement entre $\mathrm{EV}=1000\left(\mathrm{KN} / \mathrm{m}^{2}\right)$ sols très déformables

$\mathrm{EV}=300.000\left(\mathrm{KN} / \mathrm{m}^{2}\right)$ sols sables et graviers très compacts [5].

D'après le CCTP minimum requis pour la vérification des remblais $\mathrm{EV} 2>50 \mathrm{MPa}$, et $\mathrm{K}<2,0$.

$$
E V_{1}=\frac{112,5}{W_{1}}, E V_{2}=\frac{90}{W_{2}}, K=\frac{E V_{2}}{E V_{1}}
$$

\section{Etude expérimentale}

La présente partie est consacrée à la caractérisation et à l'analyse de quelques matériaux, qui peuvent être utilisés, pour l'exécution des remblais des chaussées, et d'envisager leur utilisation dans les différentes sections $\mathrm{du}$ projet, de l'autoroute Est/Ouest algérien, conformément au plan du mouvement des terres, localisées entre Constantine - Annaba, dont il a été procédé une investigation pour localiser les zones d'emprunt, les étapes suivantes ont été effectuées :

- visite pour visualiser les lieux ;

- prendre des prélèvements pour l'identification au laboratoire ;

- classification des matériaux dans la mesure où les matériaux ont présenté des bonnes caractéristiques ;

- l'exécution des remblais.

\subsection{Résultats des essais au laboratoire}

\subsubsection{Les essais d'identification}

Des essais préliminaires (Teneur en eau, analyse granulométrique, Limite de liquidité, limite de plasticité, Indice de plasticité, Indice de consistance, Valeur de bleu), ont été effectués en laboratoire pour la détermination des différentes caractéristiques des échantillons, afin de définir une classification des matériaux utilisables dans la réalisation des remblais et des couches de forme, A partir de résultats. On remarque que cet échantillon est composé d'éléments suivants :

- cailloux (39\%) de diamètre 200 à $20 \mathrm{~mm}$;

- gravillons (38\%) de diamètre 20 à $2 \mathrm{~mm}$;

Et la fraction $0-50 \mathrm{~mm}$ contenus dans cet échantillon est $84 \%$. Selon le GTR [6], et la norme AFNOR NF P 11300 [7], la classe de ce matériau (prévenance S3 EMP4 au PK : 291+000 à une profondeur 0 à $5 \mathrm{~m}$ ) est classé dans $\mathrm{C}_{1} \mathrm{~B}_{5 \mathrm{~S}}$, (sols comportant des fines et des gros éléments et cailloux anguleux, peu charpentes avec une importante fraction sableuse).

\subsubsection{Essais d'état (mécanique d'identification)}

\subsubsection{Essai Proctor}

Afin d'étudier la variation de $\rho \mathrm{d}=\mathrm{f}(\mathrm{w})$ et de déterminer $\mathrm{w}_{\text {opt }}$ et $\rho d$ correspondant, on a effectué l'essai Proctor, les résultats obtenus sont présentés sous formes de figure 2 .

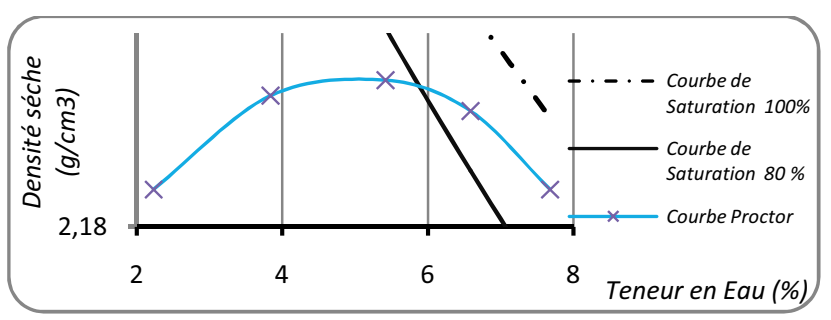

Fig. 2. Courbe d'essai Proctor.

\subsubsection{Essai de cisaillement rectiligne à la boite}

Afin d'étudier la valeur de l'angle de frottement et la cohésion, des essais mécanique (cisaillement rectiligne) ont été effectués, les résultats obtenus sont représenté sur la figure 3. 


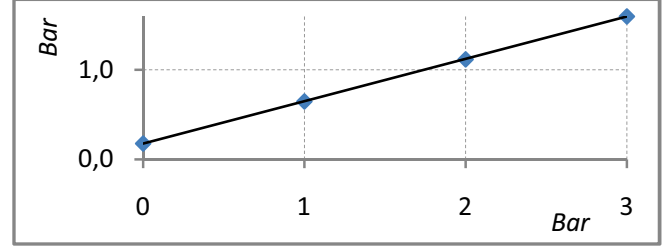

Fig. 3. Courbe intrinsèque.

D'après la figure 3 , on peut tirer l'angle de frottement de l'ordre de $25,41^{\circ}$ et la cohésion de 0,18 bar.

\subsection{Contrôle de qualité de compactage IN Situ}

\subsubsection{Essai Gammadensimètre}

Dans le but de contrôler les densités sèches et la teneur en eau en place (Contrôle des compacités lors d'exécution des remblais), des mesures ont été faites à l'aide du Gammadensimètre. Les résultats obtenus, Selon le GTR [6], et CCTP la valeur du report $\mathrm{DC} \geq 97 \%$ popt, donc l'exécution de la couche du remblai est bonne, reste la vérification de la portance du remblai au moyen de l'essai de plaque.

\subsubsection{Essai de plaque}

Dans le but de déterminer le coefficient K (EV2/EV1), on a effectué l'essai de plaque qu'est fondé sur deux cycles de chargement et déchargement, permettant la détermination de EV1, EV2, le Module sous chargement statique à la plaque,

Afin de valider nos résultats expérimentaux obtenus, il a été jugé nécessaire de les comparer d'une part, avec les résultats obtenus, à partir des méthodes théoriques, et d'autre part avec les résultats obtenus numériquement.

\section{Modélisation par éléments finis}

\subsection{Maillage}

On règle la finesse du maillage présenté sur la figure 4 , et le type de maillage utilisé pour les calculs. Il est constitué d'éléments quadratiques et bien raffiné autour de la plaque. Le modèle axisymétrie de référence se fait par des éléments à 15 nœuds. La plaque est modélisée avec
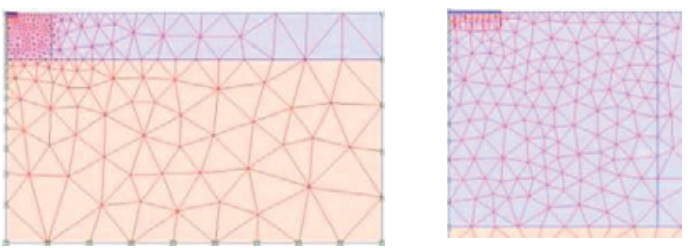

plate [8].

Fig. 4. Maillage du modèle :(a) vue d'ensemble, (b) le pourtour de la plaque.

\subsection{Présentation des résultats et analyse}

La totalité du calcul se déroule dans le domaine élastique. Les résultats de la modélisation numérique sont montrés sur la figure 5, en comparaison avec les mesures obtenus expérimentalement.

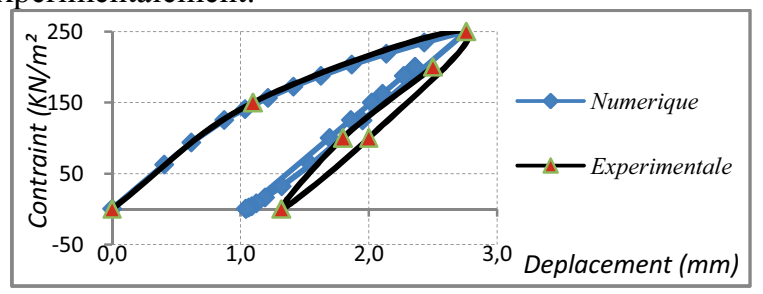

Fig. 5. Résultat de la modélisation de l'essai de chargement.

\subsection{Etude paramétrique}

\subsubsection{Cohésion c}

Les valeurs du module sous chargement statique à la plaque EV1, EV2 et le rapport K (EV2/EV1) obtenus numériquement à l'aide de code Plaxis et avec le model Mohr Coulomb, sous l'influence de la variation de cohésion $\mathrm{C}$, sont regroupés avec les mesures obtenues expérimentalement dans le tableau 1.

Table 1. Résultats des essais de plaque avec la variation de la cohésion C.

\begin{tabular}{ccccccc}
\hline \multirow{2}{*}{ Paramètres } & $\begin{array}{c}\Phi \\
\text { deg }\end{array}$ & $\begin{array}{c}\mathrm{W}_{1} \\
\mathrm{~mm}\end{array}$ & $\begin{array}{c}\mathrm{W}_{2} \\
\mathrm{~mm}\end{array}$ & $\begin{array}{c}\mathrm{EV}_{1} \\
\mathrm{MPa}\end{array}$ & $\begin{array}{c}\mathrm{EV}_{2} \\
\mathrm{MPa}\end{array}$ & $\mathrm{K}$ \\
\hline \hline \multirow{4}{*}{ Numérique } & 40 & 1,67 & 1,33 & 67,426 & 67,918 & 1,007 \\
& 30 & 1,8 & 1,33 & 62,553 & 67,918 & 1,086 \\
& 20 & 2,24 & 1,33 & 50,269 & 67,92 & 1,351 \\
& 15 & 2,72 & 1,33 & 41,391 & 67,676 & 1,635 \\
\hline Experimental & & 2,76 & 1,34 & 40,761 & 67,164 & 1,648 \\
\hline
\end{tabular}

Les variations du module sous chargement statique à la plaque EV2 et le rapport K (EV2/EV1) avec la cohésion sont présentes dans la figure 6 .

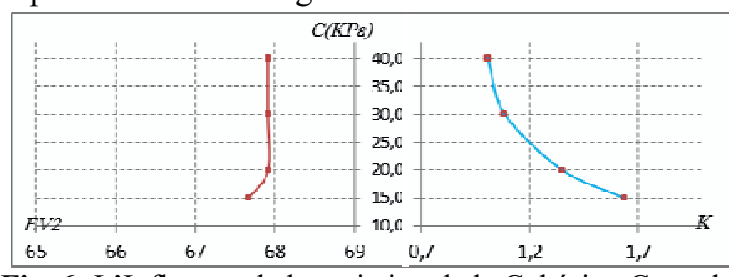

Fig. 6. L'Influence de la variation de la Cohésion C sur le module sous chargement statique à la plaque EV2 et le rapport $\mathrm{K}(\mathrm{EV} 2 / \mathrm{EV} 1)$.

D'après les résultats obtenus au figure 6 , on constate que l'influence de la cohésion sur le module EV2 et presque négligeable c'est-à-dire lorsque la cohésion $\mathrm{C}$ augmente le Module sous chargement statique à la plaque EV2 devient constant. Par contre l'influence de la cohésion sur le rapport $\mathrm{K} \quad(\mathrm{EV} 2 / \mathrm{EV} 1)$ devient considérable car lorsque la cohésion $\mathrm{C}$ augmente le rapport $\mathrm{K}$ diminue.

Les résultats obtenus sont en accord avec l'effet de l'augmentation de la cohésion sur le comportement du remblai (plus de cohésion donne plus de résistance) le module sous charge statique augment EV1 et le rapport K (EV2/EV1) $\mathrm{K}<2$, donc un bon compactage.

\subsubsection{L'Angle de frottement $\varphi$}


On présente ci-dessous les résultats de la modélisation numérique pour l'essai de plaque, que l'on compare aux mesures obtenues expérimentalement. La figure 7, représente le déplacement vertical en fonction de la charge appliquée pour les deux cycles de chargement et déchargement (NF P94-117-1).

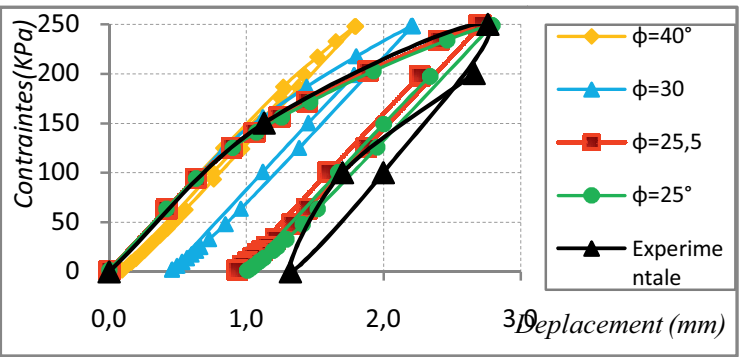

Fig. 7. Courbe charge déplacement vertical de la modélisation de l'essai de plaque.

Les déplacements calculés par le modèle de MohrCoulomb comme on a pu le constater sur le figure 7 , lorsque l'angle de frottement augmente, l'élargissement de la courbe diminue, donc on observe l'évolution inverse de déplacements, On note également que pour l'essai $\mathrm{N}^{\circ} 123$, la simulation avec le modèle de MohrCoulomb donne des déplacements très proches des mesures expérimentales. Les résultats obtenus sont montrés sur la figure 8 .

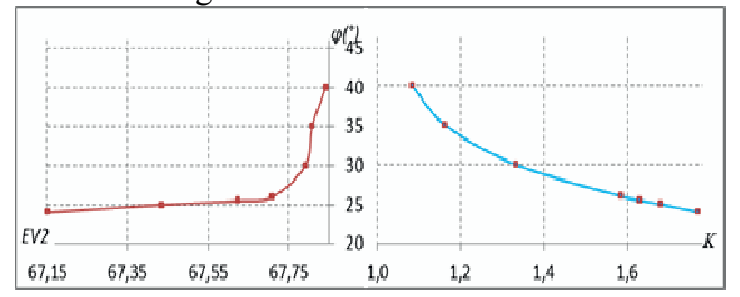

Fig. 8. L'Influence de la variation de l'angle de frottement $\varphi$ sur le module sous chargement statique à la plaque EV2 et le rapport $\mathrm{K}(\mathrm{EV} 2 / \mathrm{EV} 1)$.

D'après la figure 8 , on constate qu'il y a une influence considérable lors de l'augmentation de l'angle de frottement, on observe que le module sous charge statique EV2 augment et l'évolution inverse de rapport $\mathrm{K}(\mathrm{EV} 2 / \mathrm{EV} 1)$, et donc on obtient un bon compactage.

\subsubsection{Module d'Young $E$}

Les résultats obtenue numériquement avec la variation du module d'Young E, sont représentés sur la figure 9.

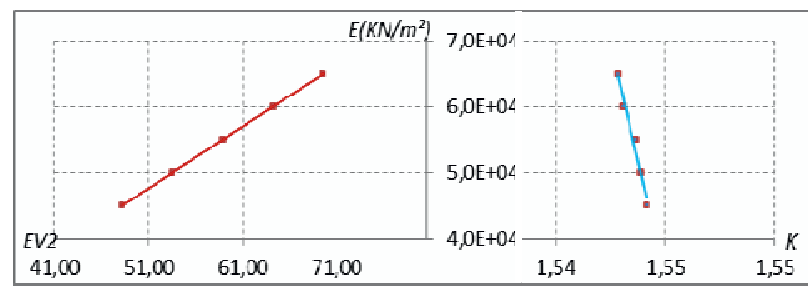

Fig. 9. L'Influence de la variation de module de d'Young E sur le module sous chargement statique à la plaque EV2 et le rapport K (EV2/EV1).

Les variations du module sous chargement statique à la plaque EV2 et le rapport K avec le Module de d'Young E suivent une loi linéaire, figure 9, Lorsque Le module
d'Young E augmente le rapport K (EV2/EV1) est constant puisque le module EV2 augmente avec l'augmentation de module sous chargement statique à la plaque EV1, c'est-à-dire EV2 et EV1 augmentent avec la même proportion.

\subsubsection{Coefficient de Poisson v}

La figure 10, représente la variation du coefficient de Poison avec le module sous chargement statique à la plaque EV2 et le rapport K (EV2/EV1).

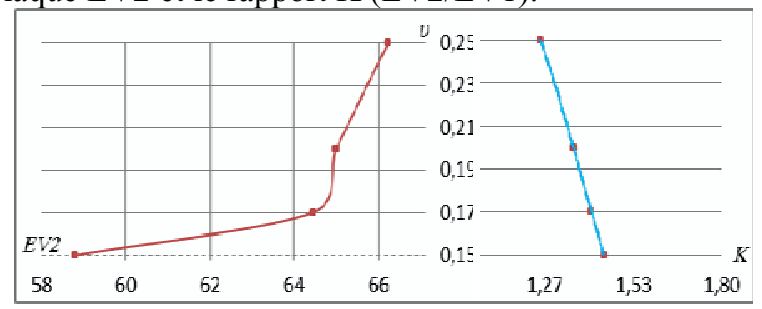

Fig. 10. L'Influence de la variation de coefficient de Poison sur le module sous chargement statique à la plaque EV2 et le rapport $\mathrm{K}(\mathrm{EV} 2 / \mathrm{EV} 1)$.

D'après la figure 10 , on constate qu'il y a une influence considérable lord de l'augmentation de coefficient de Poison, il provoque une augmentation de module sous charge statique EV2 et l'évolution inverse de rapport $\mathrm{K}$ (EV2/EV1), et donc un bon compactage.

\subsubsection{Coefficient de pression des terres au repos $K_{0}$}

La figure 11 représente l'influence de la valeur du coefficient de pression des terres au repos.

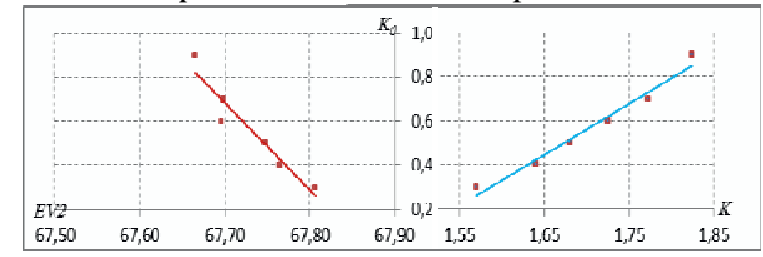

Fig. 11. L'Influence de la variation de coefficient de pression des terres au repos $\mathrm{K}_{0}$ sur le module sous chargement statique à la plaque EV2 et le rapport K (EV2/EV1).

D'après les résultats obtenus au figure 11 , on constate que l'influence de coefficient de pression des terres au repos $\mathrm{K}_{0}$ sur le Module EV2 et presque négligeable, c'est-à-dire lorsque le coefficient K0 augmente, le module EV2 devient constant. Par contre l'influence de coefficient $\mathrm{K}_{0}$ sur le rapport $\mathrm{K}$ (EV2/EV1) devient considérable car lorsque le coefficient $\mathrm{K}_{0}$ augmente le rapport $\mathrm{K}$ augmente.

\subsection{Paramètres géométriques du remblai}

On a étudié l'influence de la hauteur $\mathrm{H}$ du remblai sur le Module sous chargement statique à la plaque EV2 et le rapport $\mathrm{K}$ (EV2/EV1). A cet effet, on a choisi deux hauteurs différentes $(1,2 \mathrm{~m}, 6 \mathrm{~m})$. Les résultats obtenus numériquement à l'aide de code Plaxis, sont représentés dans le tableau 2.

Table 2. L'influence de hauteur du remblai.

\begin{tabular}{llllllll}
\hline Paramètres & $H$ & $\Phi$ & $\mathrm{W}_{1}$ & $\mathrm{~W}_{2}$ & $\mathrm{EV}_{1}$ & $\mathrm{EV}_{2}$ & $\mathrm{~K}$ \\
\hline \hline
\end{tabular}




\begin{tabular}{cccccccc}
\hline & $\mathrm{m}$ & $\mathrm{deg}$ & $\mathrm{mm}$ & $\mathrm{mm}$ & $\mathrm{MPa}$ & $\mathrm{MPa}$ & \\
\hline \hline \multirow{2}{*}{ Numérique } & 6 & 24 & 3,43 & 1,54 & $\mathbf{3 2 , 8 5}$ & $\mathbf{5 8 , 3 6}$ & $\mathbf{1 , 7 7 7}$ \\
& 1,2 & 24 & 2,75 & 1,33 & $\mathbf{4 0 , 9 5}$ & $\mathbf{6 7 , 6 8}$ & $\mathbf{1 , 6 5 3}$ \\
\hline
\end{tabular}

D'après les résultats obtenus au tableau 2, il est clair que lorsque la hauteur augmente le rapport K (EV2/EV1) augmente, ce qui signifie que le bon compactage est obtenu pour des remblais qui ont une faible épaisseur.

\subsection{L'Ecart entre la simulation numérique et expérimentale}

Afin de valider l'étude numérique avec l'expérimentale, une étude comparative entre les résultats obtenus a été effectuée. Les résultats sont représentés dans le tableau 3.

Table 3. L'Ecart entre la simulation numérique et expérimentale.

\begin{tabular}{lccccc}
\hline Paramètres & $\begin{array}{c}\mathrm{W}_{1} \\
\mathrm{~mm}\end{array}$ & $\begin{array}{c}\mathrm{W}_{2} \\
\mathrm{~mm}\end{array}$ & $\begin{array}{c}\mathrm{EV}_{1} \\
\mathrm{MPa}\end{array}$ & $\begin{array}{c}\mathrm{EV}_{2} \\
\mathrm{MPa}\end{array}$ & $\mathrm{K}$ \\
\hline \hline Numérique & 2,79 & 1,33 & 40,317 & 67,436 & 1,680 \\
\hline Experimental & 2,76 & 1,34 & 40,761 & 67,164 & 1,648 \\
\hline Ecart (\%) & 1,8 & 0,68 & 1,83 & 0,68 & 1,12 \\
\hline
\end{tabular}

D'après ces résultats, on constate une compatibilité entre les résultats de la modélisation numérique et les résultats obtenus expérimentalement.

\section{Conclusions}

Les principales conclusions qui peuvent être tirées de cette étude sont les suivantes :

- Les résultats obtenus sont en accord avec l'effet de l'augmentation de la cohésion sur le comportement du remblai (plus de cohésion donne plus de résistance) le module sous charge statique EV1 augment et le rapport $\mathrm{K}(\mathrm{EV} 2 / \mathrm{EV} 1) \mathrm{K}<2$, donc un bon compactage.

- L'influence est considérable lors de l'augmentation de l'angle de frottement, on observe que le module sous charge statique EV2 augment et l'évolution inverse de rapport $\mathrm{K}(\mathrm{EV} 2 / \mathrm{EV} 1)$, et donc on obtient un bon compactage.

- Lorsque Le module d'Young E augment le rapport K (EV2/EV1) est constant puisque le module sous chargement statique à la plaque EV2 augment avec l'augmentation du module sous chargement statique à la plaque EV1, c'est-à-dire EV2 et EV1 augmentent avec la même proportion.

- lorsque la hauteur du remblai augmente le rapport $\mathrm{K}$ (EV2/EV1) augmente, ce qui signifier que le bon compactage est obtenu pour des remblais qui ont une faible épaisseur.

- La validation du modèle numérique, avec des résultats expérimentaux (une compatibilité), et l'établissement d'un modèle numérique réel présentatif d'un sol, donnent des résultats nettement plus satisfaisants avec le modèle de Mohr-Coulomb pour les paramètres mécaniques à savoir l'angle de frottement interne $\varphi=24^{\circ}$, cohésion $\mathrm{C}=15,5 \mathrm{KPa}$, module d'Young $\mathrm{E}=6 \mathrm{E}+4 \mathrm{MPa}$ et coefficient de poison $v=0,3$ (bonne estimation des déplacements et bonne allure générale de la déformée).
- Les résultats de la modélisation numérique des essais de plaque sur les remblais de l'autoroute Est/Ouest sont globalement très satisfaisants. Ces résultats encourageants demandent à être confirmés pour d'autres types de sol.

\section{References}

1. G. JEUFFROY, R. SAUTEREY- Cour des routes assises des chaussées, (1985).

2. B. Dubisson- Encyclopedie pratique de la construction et du batiment tome III Travaux publics, Libraire Aristide Quillet PARIS (VIIe).

3. NORME : Mesure du module sous chargement statique à la plaque ( $\left.E V_{2}\right)$ NF P 94-117-1, éditée et diffusée par 1 'association française de normalisation (afnor), tour Europe 92049 paris la défense Cedex, avril (2000).

4. S. Boujlala, Relation entre l'essai de plaque ME et l'essai dynamique $B \& C$, Ecole d'ingénieur et d'architectures de Fribourg, (2007).

5. HABIBALLAH Taha El Mokhtar, modélisation des déformations permanentes des graves non traitées, thèse de doctorat, département du génie civil, université de LIMOGES.

6. SETRA, LCPC- Guide technique, Réalisation des remblais et des couches de forme, Fascicule II : Annexe techniques, 2eme Edition, (2000)

7. NORME : Exécution des terrassements NF P 11-300, éditée et diffusée par 1'association française de normalisation (afnor), tour Europe cedex 792049 paris la défense.

8. R.B.J. Brinkgereve, P.A. Vermeer, PLAXIS version 8 , scientific manual, DELFT University of Technology and PLAXIS BV, Pays-Bas, (2003). 